\section{Estimulação psicossocial e plasticidade cerebral em desnutridos}

\section{Psychosocial stimulation and brain plasticity in malnourished individuals}

\begin{abstract}
Evidences from studies in animal models and human beings are reviewed, showing the benefits produced by programed sensorial and psychosocial stimulation on neuro-behavioral functions of early malnourished individuals. It is emphasized that brain plasticity might play a fundamental role in this process. It is showed also that recent electrophysiological experiments - by using the cortical spreading depression technique - as well as observational studies in children indicate that brain regions may have a differential participation in the recovering of the brain and behavioral functions damaged by malnutrition. A new health care approach, taking into account that regional differences for treatment of children suffering early malnutrition, is suggested.
\end{abstract}

Key words Nutrition disorders, Neuronal plasticity, Environmental enrichment
Jailma Santos-Monteiro 1

Rubem Carlos de Araújo Guedes 2

Raul Manhães de Castro 3

José Eulálio Cabral Filho 4

1-3 Departamento de Nutrição. Centro de Ciências da Saúde. Universidade Federal de Pernambuco. Rua Professor Nelson Chaves, s. n. Campus Universitário. Recife, Pernambuco, Brasil, CEP: 50.670-901

4 Instituto Materno Infantil de Pernambuco, IMIP

Resumo É feita uma revisão sobre as estratégias e efeitos da estimulação sensorial e ambiental de indivíduos desnutridos. Reportam os autores evidências provenientes de experimentos com modelos animais e de estudos em seres humanos, mostrando os beneficios da administração da estimulação sensorial ou psicossocial programadas sobre as funções neurocomportamentais. Mostram ainda a importante participação que a plasticidade cerebral pode ter neste processo. Finalmente enfatizam que as evidências eletrofisiológicas - obtidas pela técnica da depressão alastrante cortial em animais - e as observações em seres humanos indicam que as regiões cerebrais comportam-se diferencialmente nesta recuperação. Daí, sugerem uma abordagem nos cuidados médicos em indivíduos desnutridos levando em conta estas peculiaridades regionais do cérebro.

Palavras-chave Desnutrição, Plasticidade cerebral, Enriquecimento ambiental 


\section{Introdução}

As evidências de que a estimulação psicossocial é capaz de promover, em grande parte, a recuperação de funções neuro-motoras e cognitivas de indivíduos previamente desnutridos são atualmente numerosas. ${ }^{1-5}$ Neste contexto também tem sido mais recentemente demonstrado que processos neurais adaptativos denominados genericamente de plasticidade cerebral.6-8 - sinaptica, neuronal e de regiões encefálicas- desempenham relevante papel nesta recuperação. Entretanto, os estudos indicam que a estimulação (quer sensorial, quer afetiva) não é suficiente, por si só, para se obter esse efeito, sendo necessária também a concomitante reabilitação nutricional daqueles indivíduos.2-4 A contribuição de autores como Grantham McGregor et al.2 que se têm dedicado ao estudo desta questão e de Rosenzweig et al. ${ }^{9}$, ou Bach-y-Rita ${ }^{10}$ que têm estudado a plasticidade cerebral, é de fundamental importância para a compreensão dos mecanismos da recuperação funcional de indivíduos desnutridos. A presente revisão abordará, as pesquisas destes e de outros autores, tratando dos aspectos nutricionais, do desenvolvimento cerebral e da estimulação psicossocial nas suas relações com os processos de plasticidade cerebral em crianças e em modelos animais previamente desnutridos.

\section{Efeito da desnutrição sobre o desenvolvimento ponderal e cerebral}

As deficiências nutricionais decorrentes da insuficiente ingestão de macro e/ou micronutrientes, levam a graus variáveis de desnutrição, reconhecidamente um grave problema mundial, principalmente nos países em desenvolvimento. Quando incide em crianças, ela constitui um problema de saúde pública, ocorrendo isoladamente ou associada a outros fatores que aumentam a morbidade e a mortalidade. 11 Os efeitos da desnutrição a longo prazo foram divulgados por Stoch e Smythe12 bem como por outros autores. ${ }^{4}$ Através de estudos seqüenciais, em que múltiplas análises foram realizadas aos cinco, dez, 15 e 20 anos com crianças que sofreram desnutrição na infância, eles verificaram alterações significantes nos seguintes parâmetros: circunferência da cabeça; altura; peso; quocientes de inteligência verbal e não verbal.

No que se refere ao sistema nervoso central, a deficiência nutricional no início da vida é mais grave, principalmente porque nesta fase o crescimento e o desenvolvimento desse sistema estão ocorrendo com grande intensidade, através dos processos de hiperplasia, hipertrofia e mielinização. A neurogênese, a gliogênese e a migração neuronal realizam-se, então, com velocidade máxima, provocando um rápido aumento do peso cerebral. Por esse motivo, essa fase é denominada de período de crescimento rápido do cérebro (PCRC). É considerado, como um dos períodos críticos mais importantes para o desenvolvimento cerebral, por ser uma etapa de grande vulnerabilidade a vários tipos de agressão, inclusive a nutricional. 13 O PCRC ocorre em épocas distintas nas diversas espécies animais. Assim, nos seres humanos inicia-se no período pré-natal (último trimestre de gestação) continuando-se até os primeiros anos de vida (dois a quatro anos); na cobaia, corresponde à vida pré-natal; na rato e no cão, ocorre no período de aleitamento.13-15

Evidências da literatura científica, indicam que todos os nutrientes estudados têm influência na maturação do cérebro. Contudo, a proteína parece ser o componente mais crítico a interferir durante o PCRC. Assim, a síntese de proteínas estruturais, o crescimento, a organização funcional e a produção de neurotransmissores cerebrais dependem da disponibilidade de aminoácidos essenciais contidos nas proteínas da dieta. ${ }^{14,16}$

Inúmeros estudos têm demonstrado uma estreita associação entre a ocorrência da desnutrição proteicocalórica grave e o prejuízo para o desenvolvimento do cérebro, com a conseqüente alteração do seu funcionamento. ${ }^{17-28}$

Em animais de laboratório, a desnutrição protéica em idades precoces provoca alterações no tecido nervoso, tais como: diminuição nos teores de glicogênio e glicose cerebrais; aumento na utilização de corpos cetônicos; aumento na concentração de ácido glutâmico e glutamina. 18,29,30 A carência nutricional imposta durante a gestação acarreta, nos filhotes, a diminuição da concentração de DNA e das proteínas cerebrais, bem como alterações das taxas de divisão celular, resultando em redução do número final de células nervosas. 15,31,32 No período de aleitamento, a desnutrição leva a uma diminuição do peso do cerebelo, do hipocampo e do córtex cerebral, evidenciando-se uma maior vulnerabilidade dessas estruturas à agressão nutricional. 33 Alterações comportamentais e eletrofisiológicas em animais desnutridos no período de gestação e aleitamento também foram demonstradas.24-26,34.

Há cerca de 30 anos se conhece que em crianças desnutridas gravemente, os teores cerebrais de DNA, 35 de gangliosídeos 36 e de cerebrosídeos 37 estão reduzidos. Foi igualmente observada a redução das dimensões do encéfalo, caracterizada através de medidas post-mortem 38 e também in vivo através de 
pneumoencefalografia. $39 \mathrm{O}$ desenvolvimento intelectual de crianças desnutridas durante os primeiros anos de vida encontra-se prejudicado, inclusive após a recuperação nutricional.3,12,24,40-49 Em registros eletroencefalográficos de crianças desnutridas precocemente foram observadas alterações caracterizadas por redução da amplitude e a lentificação das ondas. 48,49

\section{Plasticidade cerebral e nutrição}

É muito freqüente a observação de que grupos de crianças oriundas de famílias de baixo nível socioeconômico apresentam, além de deficiência ponderoestatural, maior incidência de retardo mental e de alterações motoras e, ainda, baixos índices de rendimento escolar, em relação a crianças bem nutridas. 50 Dados da literatura sugerem que o retardo do crescimento e da maturação somática de crianças são uma das consequiências mais graves da desnutrição crônica. Entretanto, não se tem a mesma certeza a respeito dos mecanismos das alterações mentais e motoras, por serem muitos os fatores ambientais, além do nutricional, que podem influir de modo negativo nestes processos.21,50-54 Na tentativa de uma melhor explanação teórica, Levitsky e Barnes, 55 divulgaram a hipótese do "isolamento funcional", na qual consideraram que as taxas diminuídas de crescimento e o atraso no desenvolvimento psicomotor em indivíduos malnutridos, se deveriam à adaptação do organismo à desnutrição precoce. Essa adaptação poderia estar envolvida com a conservação de energia, de modo que o indivíduo utilizasse melhor sua quota energética, dando prioridade à manutenção corporal; em segundo lugar, ao crescimento e por último a alguma atividade comportamental ou cognitiva. Dessa forma, a energia necessária para a sobrevivência seria poupada, apresentando esse indivíduo, além de reduzidos tamanho e peso corporal, exploração diminuída do ambiente físico e social, em relação à idade (ou seja: curiosidade diminuída; habilidades motoras e intelectuais correspondentes à de um organismo mais jovem).

Esses e outros autores mostraram que algumas alterações neurais, ocasionadas pela desnutrição podem ser parcialmente revertidas (ou compensadas) pelo tratamento de estimulação ambiental, empregado durante o processo de desnutrição, ou mais tarde, durante o período de recuperação alimentar. A partir desses resultados, os autores inferiram que a estimulação ambiental é uma condição que pode ajudar a prevenir ou recuperar os efeitos prejudiciais da desnutrição, sobre alguns parâmetros da função cerebral, tanto em animais de laboratório54-56 como em seres humanos.2,3
A recuperação parcial ou total de funções cerebrais, após recuperação nutricional associada à estimulação psicomotora e sensorial, é um dos exemplos relacionados à capacidade adaptativa (ou plástica) do tecido nervoso frente a situações adversas. Considerando-se que a interação de um indivíduo com o seu ambiente é feita através dos seus órgãos dos sentidos, podemos então entender que os estímulos sensoriais provenientes do ambiente exercem influência decisiva sobre o sistema nervoso, sobretudo durante o período de desenvolvimento rápido do encéfalo. 57 Contudo, para uma melhor compreensão acerca de como os estímulos sensoriais podem influenciar o desenvolvimento de estruturas cerebrais, é necessário expormos um breve histórico das evidências que culminaram com o conceito de plasticidade cerebral. São clássicas as observações clínicas e anatomo-patológicas feitas em indivíduos acometidos por lesões cerebrais publicadas em 1861 por Broca, citado por Kandel et al. (1995: 631). ${ }^{58}$ Esse autor constatou que havia uma correlação estreita entre as áreas cerebrais lesadas e a perda de determinadas funções e particularmente da fala. Tais evidências serviram de firme suporte para a hipótese da chamada "localização funcional" no cérebro. Os defensores dessa hipótese propunham que cada função cerebral estava estritamente relacionada a uma área do cérebro e que, uma vez lesada determinada área, não mais haveria possibilidade de recuperação da função perdida. Contudo, observações clínicas de recuperação parcial de funções em pacientes com danos cerebrais já eram, desde essa época, reconhecidas. Esses dados se contrapunham portanto às hipóteses "localizacionistas" predominantes oriundas das pesquisas de Broca e, assim, os estudos sobre a recuperação de funções cerebrais foram por muito tempo ignorados. Não obstante, réplicas experimentais das evidências clínicas acima citadas foram realizadas em animais de laboratório, verificando-se finalmente que o tecido cerebral lesado pode modificar-se ao longo do tempo recuperando funções perdidas. 10

A partir desses achados foi estabelecido o conceito de plasticidade cerebral que aliás já tinha sido proposto teoricamente por Hebb em 1949, citado por Rosenzweig e Bennett, (1996: 57)59 definida como sendo a capacidade de o tecido cerebral modificar sua organização e função frente a distúrbios patológicos ou a lesões precoces desse órgão ou, contrariamente, em consequiência da ação de estímulos ambientais. 10,54,60-70

As modificações no desenvolvimento cerebral concernentes à função plástica do tecido nervoso têm sido demonstradas em animais de laboratório criados em ambientes com diferentes graus de complexi- 
dade. Quando esses animais são submetidos, durante o seu desenvolvimento, a um ambiente "rico" em estímulos e/ou a sessões de estimulação ambiental (procedimento esse denominado também enriquecimento ambiental), é evidenciado aumento no peso e espessura de estruturas corticais e subcorticais; no teor de aminas cerebrais; na atividade de enzimas neuronais; no número e diâmetro dos capilares corticais e diminuição na densidade celular, em comparação com animais criados em ambientes "pobres" em estímulos. O aumento do número de contatos sinápticos e de ramificações dendríticas encontrados nesses animais, evidenciam também plasticidade sináptica.9,53,71-74 Tais alterações, ocasionadas pela manipulação ambiental, são mais bem evidenciadas quando ela é imposta durante as fases iniciais da vida, uma vez que o tecido cerebral está mais susceptível a transformações. ${ }^{53,73}$ Animais criados em ambientes com poucos estímulos ou em condições de isolamento, apresentam alterações cerebrais geralmente opostas àquelas encontradas em animais estimulados. 53,73 Igualmente, alterações comportamentais associadas à estimulação ambiental ou ao isolamento têm sido descritas. 17,55,75-79.

\section{Tratamento clínico de crianças com desnutrição precoce: utilização da estimulação psicossensorial e motora associada à recuperação nutricional}

Algumas hipóteses têm sido consideradas com a finalidade de explicar o mecanismo pelo qual a desnutrição precoce e a estimulação ambiental podem interagir para produzir mudanças no comportamento. Uma das hipóteses é a já mencionada por Levitsky e Barnes, 55 em relação ao isolamento funcional dos desnutridos. Tal hipótese postula que a desnutrição poderia torna-lo fisiologicamente menos capaz de receber informações do ambiente e interagir com este, ou que a desnutrição produziria per se comportamentos incompatíveis com a informação proveniente do meio externo. A estimulação ambiental teria então a finalidade de ajudar o indivíduo desnutrido a se adaptar funcionalmente melhor, para romper o referido isolamento funcional. Também já foi mostrado que a ação da estimulação psicossocial sobre a cognição (escala de inteligência de Weschler, testes verbais e de raciocínio) de crianças previamente desnutridas tem um efeito duradouro, podendo estender-se por vários anos. 4

No caso de estudos com crianças, é necessário considerar que a desnutrição não pode ser analisada como único fator etiológico do isolamento funcional, mas, deve ser incluída num conjunto de outras va- riáveis sociais e econômicas. Tem-se até dado mais atenção a outros fatores ambientais, além dos nutricionais que influem sobre a saúde física e mental de crianças que padecem de desnutrição. 19 Assim é muito freqüente a observação de baixos índices de rendimento escolar e deficientes desenvolvimentos mental e motor em crianças de famílias de baixa renda. Embora, seja difícil identificar com precisão o grau de interferência das diversas variáveis (tipo de deficiência nutricional, se protéica ou calórica; interações sociais mãe-filho, criança-criança, criança-adulto e a qualidade dos estímulos ambientais), sobre o comportamento infantil, uma vez que essas variáveis se encontram geralmente associadas, vários autores tem estudado o assunto. Assim, o comportamento lúdico, que é extremamente dependente das interações do microambiente familiar, foi mostrado estar prejudicado quando a desnutrição esta associada a condições socioeconômicas precárias. .0-83 $^{8}$

Barret e Radke-Yarrow ${ }^{1}$ estudaram os efeitos comportamentais, a longo prazo (seis anos depois), de períodos de déficit nutricional precoce de duas diferentes suplementações alimentares (protéicocalórica e apenas calórica). As avaliações comportamentais, feitas à idade de seis a oito anos de vida mostraram que as crianças tratadas com suplementação protéica apresentaram mais interesse em explorar o ambiente novo, mais envolvimento em jogos competitivos, maior persistência em tarefas frustrantes, melhor controle do impulso motor, maior iniciativa em tarefas grupais, mais afetividade com outras crianças, melhor nível de envolvimento social e de atividade motora, além de menor nível de ansiedade e menos timidez, em comparação com crianças que receberam suplementação apenas calórica. Esses mesmos indivíduos na adolescência (idade de 13 a 19 anos), foram submetidos a testes psicoeducacionais, em um estudo feito por Pollitt et al.21 Os sujeitos que receberam a suplementação protéica na infância, apresentaram pontuações mais altas de conhecimento, habilidade com números, leitura e vocabulário, bem como um menor tempo de reação à respostas aos testes, em relação àqueles que receberam suplementação calórica.

A melhora de parâmetros comportamentais, em crianças desnutridas precocemente, se dá de maneira mais evidente quando elas são submetidas à programas bem definidos de estimulação psicossensorial e motora (EPSM), associada à reabilitação nutricional. Os dados obtidos por Grantham-McGregor et al.2 reforçam essa afirmação. Esses autores encontraram efeitos benéficos da suplementação nutricional associada ou não à EPSM, sobre o desenvolvimento mental de crianças de baixo peso à idade de 9-24 
meses de vida, em Kingston, Jamaica. Os efeitos dos dois tratamentos (nutricional e da estimulação) foram aditivos, uma vez que a combinação dessas duas intervenções influenciou mais significantemente os parâmetros estudados que quando empregadas sozinhas. Em outro estudo, Grantham-MacGregor et al. 52 quantificaram uma melhora no comportamento de crianças hospitalizadas com desnutrição grave e submetidas a um tratamento de estimulação psicossocial, durante a reabilitação nutricional. Tais crianças, ao final do tratamento, não mais apresentaram apatia, hipoatividade e pouca exploração do ambiente e dos brinquedos, como no início do tratamento, tendo então, comportamento final semelhante ao das crianças controle (hospitalizadas, sem desnutrição e submetidas a estimulação). Semelhantemente, Colombo et al. ${ }^{3}$ mostraram que a reabilitação nutricional associada a EPMS, foi benéfica em produzir melhoras significativas na coordenação motora e na linguagem, bem como no desenvolvimento social e motor de lactentes com desnutrição grave. Curiosamente, as crianças que apresentaram um maior retardo psicomotor inicial foram as que obtiveram maiores benefícios com essa intervenção.

Com a finalidade de conhecer com mais clareza as relações acima citadas, Radrigan et al. 50 estudaram a relação entre o ambiente, o estado nutricional e o desenvolvimento intelectual de crianças pré-escolares (nos dois primeiros anos de vida), com desnutrição leve. Os testes diagnósticos empregados apontaram níveis de privação ambiental elevados e de estimulação psicossensorial insuficientes e inadequados. Em 73\% dessas crianças foi constatado retardo do desenvolvimento psicomotor, e em $54 \%$ as pontuações do nível intelectual estavam abaixo do normal. As correlações feitas entre as diversas variáveis, mostraram que a desnutrição precoce afetou especialmente os aspectos cognitivos (baixos níveis intelectuais) enquanto que a privação sensório-afetiva e cultural afetou o desenvolvimento psicológico de um modo geral (níveis intelectuais e desenvolvimento psicomotor).

Os resultados desses trabalhos mostram claramente os efeitos benéficos da EPSM na recuperação de crianças desnutridas. Resta esclarecer quais são os estímulos mais eficientes em produzir os efeitos obtidos e durante quanto tempo esses estímulos devem ser empregados.

Nesse sentido, o Departamento de Nutrição da Universidade Federal de Pernambuco vem desenvolvendo estudos sobre desnutrição e plasticidade cerebral com o intuito de obter subsídios que ajudem a compreender como a plasticidade do tecido nervoso se comporta frente à desnutrição. Dentro dessa linha de pesquisa, tem sido utilizado o modelo da depressão alastrante da atividade elétrica cortical (DAC) como um indicador para avaliar o estado funcional do sistema nervoso em modelos animais. $\mathrm{O}$ fenômeno consiste numa resposta reversível do tecido cortical, provocada por uma estimulação elétrica, mecânica ou química, de um ponto desse tecido. Caracteriza-se por uma diminuição (depressão) acentuada da atividade elétrica espontânea do ponto cortical submetido à estimulação e que se propaga, a partir daí, de forma concêntrica por todo o córtex (com velocidade da ordem de 2 a $5 \mathrm{~mm} / \mathrm{min}$ ). 79,80

Dentro deste enfoque uma série de experimentos foi realizada com o objetivo de averiguar a influência da estimulação sensorial sobre a DAC em animais previamente desnutridos.

Para isto ratos bem nutridos ou desnutridos foram submetidos a diversos graus de estimulação sensorial, durante períodos precoces de suas vidas. Interessantemente, observou-se que apenas os animais desnutridos, mantidos em condições de ambientes ricos em estímulos, desde a idade pré-desmame até a idade adulta (dos 7 aos 90-110 dias de idade), apresentaram redução da velocidade de propagação da DAC. Os grupos desnutridos e submetidos à estimulação ambiental após o desmame, ao contrário, não pareceram se beneficiar dessa estimulação. 79

Esses e outros experimentos sugeriram existir um efeito diferencial da estimulação ambiental sobre a DAC, dependendo do estado nutricional e do período em que a estimulação se inicia. ${ }^{60,73}$

Achados de outros autores mostraram que em animais normais, dentre as distintas áreas corticais estudadas, o córtex occipital é a região mais afetada pelo tratamento ambiental, mesmo quando esse é empregado em um período pós-desmame.60,73 De acordo com essa informação, procurou-se verificar a possibilidade de diferenças na velocidade de propagação da DAC entre distintas regiões corticais de animais normais, submetidos ou não à estimulação ambiental. Para isso, foram realizados registros eletrofisiológicos em duas áreas do córtex: a parietal e a occipital. Os dados obtidos mostraram que as velocidades de propagação da DAC foram significantemente mais baixas na região occipital, em comparação com as velocidades obtidas na região parietal, em ratos estimulados precocemente. Esses resultados confirmam que os padrões de propagação da DAC possam ser alterados, de forma mais intensa no córtex occipital, em decorrência do tratamento ambiental. 80 A evidência de que a estimulação sensorial afeta diferencialmente processos eletrofisiológicos corticais 75 permite portanto detectar mecanismos 
cerebrais subjacentes à interação nutrição-cérebroambiente e ao mesmo tempo vislumbrar possíveis explicações para esta interessante fenomenologia.

\section{Conclusões}

A pletora de trabalhos científicos sobre esta temática, realizada nas últimas décadas, demonstra portanto os benefícios que o enriquecimento ambiental pode trazer para as pessoas - em especial as crianças - que tenham sofrido de desnutrição precoce. Isto nos permite ter, hoje, grande otimismo quanto à possibilidade de sua reabilitação psiconeurológica. A mais recente compreensão dos mecanismos neurais que estão na base desta recuperação, isto é, os processos adaptativos de plasticidade do sistema nervoso, dão suporte para que sejam implementadas estratégias que ajudem a recuperação psicossocial de indivíduos ou pequenos grupos de indivíduos anteriormente desnutridos, e portanto para sua inserção em ambientes mais afluentes do que aqueles de que vieram. Assim, a investigação dos mecanismos pelos quais a neuroplasticidade pode facilitar a recuperação de desnutridos é fundamental porque o trata- mento destes indivíduos poderá ser mais adequadamente direcionado. $\mathrm{O}$ fato de diferentes regiões cerebrais serem mais ou menos susceptíveis à estimulação psicossensorial 75 certamente permitirá condutas específicas para a obtenção de melhores resultados terapêuticos. É digno de nota que estudos comportamentais em crianças vêm dar suporte aos achados laboratoriais. De fato já foi mostrado ${ }^{3}$ que crianças previamente desnutridas, mesmo recebendo posteriormente suplementação nutricional adequada e estimulação psicossocial, apresentam recuperação de alguns comportamentos, mas não de outros. Portanto é por demais desejável continuar-se buscando esclarecer os aspectos psicobiológicos subjacentes a estes complexos processos.

Apesar destas evidências e perspectivas, seria praticamente impossível atingir, com os métodos de estimulação, largas faixas populacionais. Ao lado do fato de que a reabilitação nem sempre é completa, isto nos leva a enfatizar a importância de buscar soluções não apenas para as deficiências psicológicas provenientes da subalimentação e das precárias condições sociais, mas para erradicar, pela base, as causas que lhes dão origem.

\section{Referências}

1. Barret DE, Radke-Yarrow M. (1985) Effects of nutritional supplementation on children's responses to novel, frustrating, and competitive situations. Am J Clin Nutr 1985; 42, 102-20.

2. Granthan-McGregor, SM, Powell, CA, Walker SP, Himes JH. Nutritional supplementation, psychosocial stimulation, and mental development of stunted children: the Jamaican study. Lancet 1991; 338: 1-5.

3. Colombo M, Lopez I, De Andraca I. Desnutrición grave precoz y desarrollo psicomotor. Efectos de un programa de rehabitación. Arch Latinoam Nutr 1993; 43: 146-50.

4. Walker SP, Grantham-Mcgregor SM, Powell CA, Chang SM. Effects of growth restriction in early childhood on growth, IQ, and cognition at 11 to 12 years and the benefits of nutritional supplementation and psychosocial stimulation. J Pediatr 2000; 137: 36-41.

5. Hall A, Khanh LNB, Son TH, Dung NQ, Lansdown RG, Dat DT, Hanh NT, Moestue H, Kohl HH, Bundy DAP. An association between chronic undernutrition and educational test scores in vietnamese children. Eur J Clin Nutr 2001; 55: 801-4.

6. Lent R. Cem bilhões de neurônios. São Paulo: Atheneu; 2001.
7. Vanhosse AM, Emery M, Jimenez L, Wincler DG. ERK activation by G-protein coupled recptors in mouse brain is receptor indentity-specific. J Biol Chem 2002; 277 : 9049-53.

8. Blasquez PM, Fujii N, Kojima J, Graybiel AM. A network representation of response probability in the striatum. Neuron 2002; 33: 973-82.

9. Rosenzweig MR, Bennett EL, Diamond MC, Wu SY, Slagle RW, Satran E. Influences of environmental complexity and visual stimulation on development of occipital cortex in rat. Brain Res 1969; 14: 427-45.

10. Bach-Y-Rita P. Brain platicity as a basis for recovery of function in humans. Neuropsychologia 1990; 28: 547 54.

11. Duncan GJ, Brooks-Gunn J, Klebanos PK. Economic deprivation and early childhood development. Child Dev 1994; 65: 296-318.

12. Stoch MB, Smythe PM. Does undernutrition during infancy inhibit brain growth and subsequent intellectual development? Arch Dis Child 1963; 38: 546-52.

13. Dobbing J. Vulnerable periods in devolping brain. In: Davison NA, Dobbing J, editors. Apllied neurochemistry. Oxford: Blackwell; 1968. p. 287-316. 
14. Morgane PJ, Miller M, Kemper T, Stern W, Forbes W, Hall R, Bronzino J, Kissane J, Hawrylewicz E, Resnick O. The effects of protein malnutrition on the developping nervous system in the rat. Neurosci Biobehav Rev 1978; 2: $137-230$

15. Morgane PJ, Austin-Lafrance RJ, Bronzino J, Tonkiss J, Diaz-Cintra S, Cintra L, Kemper T, Galler JR. Prenatal malnutrition and development of the brain. Neurosci Biobehav Rev 1993; 17: 91-128.

16. Wiggins RC, Fuller G, Enna SJ. Udernutrition and development of brain neurotransmitter systems. Life Sci 1984; 35: 2085-94.

17. Ballabriga A. Some aspects of clinical and biochemical changes related to nutrition during brain development in humans. In: Evrard P, Minkowski A. Developmental neurobiology. New York: Raven; 1989. p. 271-86.

18. Colombo M, Parra De La A, Lopez I. Intellectual and physical outcome of children undernourished in early life is influenced by later environmental conditions. Dev Med Child Neurol 1992; 34: 611-22.

19. Dixon SD, Levine RA, Brazelton TB. Malnutrition: a closer look at the problem in an East African village. Dev Med Child Neurol 1992; 2: 670-85.

20. Morgane PJ, Austin-Lafrance RJ, Bronzino, JD, Tonkiss J, Galler JR. Malnutrition and developing central nervous system. In: Isaacson RL, Jensen KF, editors. The vulnerable brain and environmental risks. New York: Plenum; 1992. p. 2-42.

21. Pollitt E, Gorman KS, Engel PL, Rivera JÁ, Martorell R. Nutrition in early life and the fulfillment of intellectual potential. Supplement: The INCAP follow-up study. J Nutr 1995; 125, 1111S-8S

22. Rao S, Karkhans L. Differential patterns of malnutrition among kindergarten children in Pune city. Indian J Med Res 1993; 98: 129-35.

23. Rasmussen KM. The influence of maternal nutrition on lactacion. Annu Rev Nutr 1992; 12: 103-17.

24. Resnick O, Miller M, Forbes W, Hall R, Kemper T, Bronzino J, Morgane PJ. Developmental protein malnutrition influences on central nervous system of the rat. Neurosci BioBehav Rev 1979; 3: 223-46.

25. Resnick O, Morgane PJ. Ontogeny of the levels of serotonin in various parts of the brain in severely protein malnourished rats. Brain Res 1984; 303: 163-70.

26. Ruiz S, Perez H, Hernandez H, Soto-Moyano R. Effect of early malnutrition on latencies of direct cortical responses evoked in the rat prefrontal cortex. Nutr Rep Int 1985 32: 533-8.

27. Rocha-de-Melo AP, Guedes RCA. Spreading depression is facilited in adult rats previously submitted to shor episodes of malnutrition during the lactation period. Braz J Med Biol Res 1997; 30: 663-9.

28. Picanço-Diniz CW, Araújo MS, Borba JMC, Guedes RCA NADPH-Diaphorase containing neurons and biocytin-labelled axon terminals in the visual cortex of adult rats malnourished during development. Nutr Neurosci 1998 1: 35-48.

29. John J, Patel MS, Telang SD. Effects of maternal dietary protein content on cerebral ketone body-metabolizing enzymes in the progeny of rats. J Nutr Biochem 1991; 2: 604-8.

30. Garriga J, Cussó R. Effect of starvation on glycogen and glucose metabolism in different areas of the rat brain
Brain Res 1992; 591: 277-82.

31. Colombo JP, Cervantes H, Kokorovic M, Pfister V, Perritaz R. Effects of different protein diets on the distribution of amino acids in plasma, liver and brain in the rat. Ann Nutr Metab 1992; 36: 23-33.

32. Zamenhof S. Depletion of individuals with "outstandingly high" cerebral development in rats subjected to prenatal undernutrition. Nutr Res 1991; 11: 687-90.

33. Fuller GN, Wiggins RC. Differential growth recovery within the brains of posnatally undernourished rats. Dev Brain Res 1984; 15: 280-4

34. Prasad VVTS. Maternal protein deficiency in rat: effects on central nervous system gangliosides and their catabolizing enzymes in the offspring. Lipids 1991; 26: 553-6.

35. Winick M, Rosso P, Wartelow J. Cellular growth of cerebrum, cerebellum and brain stem in normal and marasmic children. Exp Neurol 1970; 26: 393-400.

36. Dickerson JWT, Jarvis J. Effect of undernutrition and subsequent reabilitation on the growth and chemical composition of cerebellum, brain system and forebrain of the rat. Proc Nutr Soc 1971; 28: 4A.

37. Fishman MA, Frensky AL, Dodge PR. Low content of cerebral lipids in infants suffering from malnutrition. Nature 1969; 22: 552-60.

38. Brown RE. Organ weight in malnutrition with special reference to brain weight. Dev Med Child Neurol 1966; 8: $512-22$.

39. Marcondes E, Lefevre AB, Machado DVM. Desenvolvimento neuropsicomotor da criança desnutrida. 1: Má nutrição protéica. Rev Bras Psiquiatr 1969; 3:173-219.

40. Stoch MB, Smythe PM. Does undernutrition during infancy inhibit brain growth and subsequent intellectual development? Arch Dis Child 1963; 38: 546-52.

41. Cabak V, Najdanvic R. Effect undernutrition in early life on physical and mental development. Arch Dis Child 1965; 40: 532-4.

42. Lloyd-Still JD, Hurwitz I, Wolff PH, Schwachman H. Intellectual development after severe malnutrition in infancy. Pediatrics 1974; 54: 306-11.

43. Cravioto J, Delicardie E. Longitudinal study of language development in severely malnourished children. In: Serban, G. Nutrition and mental functions. New York: Plenum; 1975.

44. NwugaVCB. Effect of severe kwashiorkor on intelectual development among Nigerian children. Am J Clin Nutr 1977; 30: 1423-30.

45. Ivanovic DM, Olivares MG, Castro CG, Ivanovic RM. Nutrition and learning in Chilean school age children: Chile's metropolitan region survey 1986-1987. Appl Nutr Invest 1996; 12: 321-8.

46. Morley R, Lucas A. Nutrition and cognitive development. Br Med Bull 1997; 53: 1: 123-34.

47. Gordon N. Nutritional and cognitive function. Brain Dev 1997; 19: 165-70.

48. Valenzuela RH, Penic JH, Macias R. Aspectos clínicos, electroencefalográficos y psicologicos en la recuperación del niño desnutrido. Gac Med Mex 1959; 89: 651-61

49. Karayadi D. Electroencephalograms, learning and malnutrition. In: Dson, R. E. Protein-calorie malnutrition. New York: Academic Press: 1975. p. 103-5.

50. Radrigan ME, Guerrero F, Gallarto I, Goitia JT, Barriga R. Medio ambiente, nutrición y desarrollo intelectual. Bo 
Med Hosp Infant 1979; 36: 711-24.

51. Celedon JM, De Andraca I. Psychomotor development during treatment of severely marasmic infants. Early Hum Dev 1979; 3: 267-75.

52. Grantham-Mcgregor S, Stewart M, Powell C. Behaviour of severely malnourished children in a Jamaican hospital. Med Dev Child Neurol 1991: 33: 706-14.

53. Fernandez V, Pascual R, Ruiz S. Early life environmental deterioration, nutrition and ontogenesis of the motor cortex in the rat: a Golgi study. Biol Neonate 1993; 64: 24553.

54. Fernandez V, Bravo H, Sanhueza M, Inzunza O. NADPH-d positive neurons in the developing somatosensory cortex of the rat: effects of early and late environmental enrichment. Dev Brain Res 1998; 107: 299-307.

55. Levitsky DA, Barnes RH. Nutritional and environmental interactions in the behavioral development of the rat: long-term effects. Science 1972; 76: 68-71.

56. Lima JG. Estudo morfológico e morfométrico do corpo caloso de ratos submetidos a diferentes tipos de dieta e à estimulação sensorial e ambiental [tese doutorado]. Ribeirão Preto: Faculdade de Medicina da Universidade de São Paulo; 1992.

57. Crutcher KA. Anatomical correlates of neuronal plasticity. In: Martinez JL, Kesner RP. Learning and memory: a biological view. San Diego: Raven Press; 1991. p. 93-146.

58. Kandel ER, Schwartz JH, Jessel TM. Essentials of neural science and behavior. Stamford: Appleton \& Lange; 1995.

59. Rosenzweig MR, Bennet EL, Psychobiology of plasticity: effects of training and experience on brain and behavior. Behav Brain Res 1996; 78: 57-65.

60. Bennett EL, Diamond MC, Krech D, Rozenzweig MR. Chemical and anatomical plasticity of brain. Science 1964; 146: 610-8.

61. Wallace P. Complex environments: effects on brain development. Science 1981; 185: 1035-7.

62. Kolb B, Whishaw Q. Plasticity in the neocortex: mechanisms underlying recovery from early brain damage. Prog Neurobiol 1989; 32: 235-76.

63. Calverley RKS, Jones DG. Contribution of dendritic spines and perforated synapses to synaptic plasticity. Brain Res Rev 1990; 15: 215-49.

64. Barlow GM, Micales B, Lyons GE, Korenberg JR. Down syndrome cell adhesion molecule is conserved in mouse and highly expressed in the adult mouse brain. Cytogenet Cell Genet 2001; 94: 155-62.

65. Zilles K. Neuronal plasticity as an adaptative property of the central nervous system. Ann Anat 1992; 174: 383 91.

66. Nudo RJ, Plautz EJ, Milliken GW. Adaptative plasticity in primate motor cortex as a consequence of behavioral experience and neuronal injury. Neuroscience 1997; 9: 1323.

67. Moser MB, Trommald M, Geland TE, Anderson P. Spatial trainning in a complex environment and isolation alter the spine distribution differently in rat CA1 pyramidal cells. J Comp Neurol 1997; 380: 373-81.

68. Buonomano DV, Merzenich MM. Cortical plasticity: from synapses to maps. Annu. Rev Neurosci. 1998; 21: 14986.

69. Torasdotter M, Metsis M, Henriksson BG, Winblad B, Mohammed AH. Environmental enrichment resuls in higher level of nerve growth factor mRNA in the rat visual cortex and hippocampus. Behav Brain Res 1998; 93: 83-90.

70. Coq J-O, Xerri C. Environmental enrichment alters organizational features of the forepaw representation in the primary somatosensory cortex of adult rats. Exp Brain Res 1998; 121: 191-204

71. Diamond MC. Extensive cortical depth measurements and neuron size increases in the cortex of environmentally enriched rats. J Comp Neurol 1967; 131: 357-64.

72. Kaad JH, Plasticity of sensory and motor maps in afult mamals. Annu. Rev. Neurosci. 1991; 14: 137-67.

73. Renner MJ, Rosenzweig RR. Enriched and impoverished environments. Effects on brain and behavior. New York: Springer; 1987.

74. Sirevaag AM, Black JE, Shafron D, Greenough WT. Direct evidence that complex experience increases capillary branching and surface area in visual cortex of young rats. Dev Brain Res 1988; 43: 299-304.

75. Kaas JH. Plasticity of sensory and motor maps in adult mamals. Annu Rev Neurosci 1991; 14: 137-67.

76. Diaz JL. Brain weights correlate with behavioral parameters in individual inbred mice housed in a common an enriched environment. Behav Neural Biol 1988; 50: 164 83.

77. Hilakivi LA, Ota M, Lister RG. Effect of isolation on brain monoamines and the behavior of mice in tests of exploration, locomotion, anxiety and behavioral "despair". Pharmacol Biochem Behav 1989; 33: 371-4.

78. Mohammed AK, Winblad B, Ebendal T, Lärkfors L. Environmental influence on behavior and nerve growth factor in the brain. Brain Res 1990; 528: 62-72.

79. Santos-Monteiro J. Desnutrição, estimulação ambiental e desenvolvimento do sistema nervoso: um estudo eletrofisiológico [dissertação mestrado]. Recife: Universidade Federal de Pernambuco; 1995.

80. Santos-Monteiro J, Teodósio NR, Guedes RCA. Long-lasting effects of early environmental stimulation on cortical spreading depression in normal and early malnourished adult rats. Nutr Neurosci 2000; 3: 29-40.

81. Pollitt E. Developmental sequel from early nutrition deficiencies: concensive and probability judgements. Am Soc Nutr Sci 2000 Suppl: 350S-3S

82. Walka H, Triana N, Jahari AB, Husaini MA, Pollity E. Ef fects of an emergy and micronutrient supplement on play behavior in undernourished children in Indonésia, Eur J Clin Nutr 2000; 54 Suppl 25: 91-106

83. Rabelo ARM. Desenvolvimento infantil e desnutrição: crescimento e modo de brincar de crianças de 4 a 18 meses internadas no IMIP [dissertação de mestrado]. Recife: Instituto Materno Infantil de Pernambuco, IMIP: 2002. 